\title{
Invariant measures of nonholonomic flows with internal degrees of freedom
}

\author{
Dmitry V Zenkov ${ }^{1}$ and Anthony M Bloch ${ }^{2}$ \\ ${ }^{1}$ Department of Mathematics, North Carolina State University, Raleigh, NC 27695, USA \\ 2 Department of Mathematics, University of Michigan, Ann Arbor, MI 48109, USA \\ E-mail: dvzenkov@unity.ncsu.edu
}

Received 12 November 2002

Published 28 July 2003

Online at stacks.iop.org/Non/16/1793

Recommended by G Morriss

\begin{abstract}
In this paper we study measure preserving flows associated with nonholonomic systems with internal degrees of freedom. Our approach reveals geometric reasons for the existence of measures in the form of an integral invariant with smooth density that depends on the internal configuration of the system.
\end{abstract}

Mathematics Subject Classification: 37J15, 37J60, 70F25

\section{Introduction}

In this paper we study conditions for existence of an invariant measure for nonholonomic systems with symmetry. If present, the invariant measure results in the absence of asymptotically stable equilibria and periodic solutions of the system under consideration and thus leads to a more Hamiltonian-like behaviour of the trajectories (see Kozlov (1985) and Arnold (1988) for further details).

Kozlov (1988) and Jovanović (1998) study nonholonomic systems without shape variables and obtain a criterion for existence of an invariant measure in terms of the structure constants of a Lie group, which is the configuration space of the mechanical system in this case, and the components of the inertia tensor of the system. The dynamics of these systems reduces to a subspace of the Lie algebra of the configuration group and the measure is in fact the standard volume on this subspace. In earlier work Blackall (1941) obtained conditions for existence of integral invariants of analytic nonholonomic systems without symmetry.

In this paper we consider smooth (but not necessarily analytic) systems with internal degrees of freedom, i.e. we assume that both the shape space and the symmetry group are nontrivial. We derive conditions for existence of an invariant measure in the form of an integral invariant whose density depends on the shape configuration of the system only. This means that this density is a function of the shape variables in a local trivialization of the reduced 
phase space of the system. Of course, the density of the integral invariant depends on the phase coordinates used. In particular, it depends on the choice of the basis in the Lie algebra of the symmetry group of the system.

Our approach shows that there are geometric reasons for a nonholonomic system to possess an invariant measure of this kind. The method we develop here gives an explicit formula for the density of the integral invariant.

This study is motivated by many interesting examples of nonholonomic systems including the Chaplygin sphere (Kozlov 1988, Chaplygin 1903) and the Routh problem (see Routh (1860) and Zenkov (1995)), as well as the so-called LR systems (see Veselov and Veselova (1988)). In particular, our approach links the existence of the invariant measure of the Chaplygin sphere with the conservation of the vertical component of the angular momentum observed in this system.

The exposition is organized as follows: in section 2 we review the theory of nonholonomic systems and discuss the role of symmetries. We also derive the reduced equations in an arbitrary (as opposed to an orthogonal) body frame and give a criterion for the nonholonomic and mechanical connections to coincide. In section 3 we briefly discuss some of the theory of measure preserving nonholonomic systems without internal degrees of freedom. Our main results are presented in section 4 . To illustrate our theory, we treat a system consisting of the three-dimensional Chaplygin sleigh coupled with an oscillator, and in addition we discuss the falling disc, the Chaplygin sphere and the Routh problem.

\section{Equations of motion of nonholonomic systems with symmetries}

In this section we briefly discuss the dynamics of nonholonomic systems with symmetries. We refer the reader to Bloch et al (1996) and Zenkov et al (1998) for a more complete exposition.

\subsection{The Lagrange-d'Alembert principle}

We now describe the equations of motion for a nonholonomic system. We confine our attention to nonholonomic constraints that are linear and homogeneous in the velocity. Accordingly, we consider a configuration space $Q$ and a distribution $\mathcal{D}$ that describes these constraints. Recall that a distribution $\mathcal{D}$ is a collection of linear subspaces of the tangent spaces of $Q$; we denote these spaces by $\mathcal{D}_{q} \subset T_{q} Q$, one for each $q \in Q$. A curve $q(t) \in Q$ will be said to satisfy the constraints if $\dot{q}(t) \in \mathcal{D}_{q(t)}$ for all $t$. This distribution will, in general, be nonintegrable; i.e. the constraints are, in general, nonholonomic.

Consider a Lagrangian $L: T Q \rightarrow \mathbb{R}$. In coordinates $q^{i}, i=1, \ldots, n$, on $Q$ with induced coordinates $\left(q^{i}, \dot{q}^{i}\right)$ for the tangent bundle, we write $L\left(q^{i}, \dot{q}^{i}\right)$. The equations of motion are given by the following Lagrange-d'Alembert principle.

Definition 2.1. The Lagrange-d'Alembert equations of motion for the system are those determined by

$$
\delta \int_{a}^{b} L\left(q^{i}, \dot{q}^{i}\right) \mathrm{d} t=0,
$$

where we choose variations $\delta q(t)$ of the curve $q(t)$ that satisfy $\delta q(a)=\delta q(b)=0$ and $\delta q(t) \in \mathcal{D}_{q(t)}$ for each $t$ where $a \leqslant t \leqslant b$.

This principle is supplemented by the condition that the curve itself satisfies the constraints. Note that we take the variation before imposing the constraints; that is, we do not impose the 
constraints on the family of curves defining the variation. This is well known to be important to obtain the correct mechanical equations (see Bloch et al (1996) for a discussion and references).

The usual arguments in the calculus of variations show that the Lagrange-d'Alembert principle is equivalent to the equations

$$
-\delta L=\left(\frac{\mathrm{d}}{\mathrm{d} t} \frac{\partial L}{\partial \dot{q}^{i}}-\frac{\partial L}{\partial q^{i}}\right) \delta q^{i}=0
$$

for all variations $\delta q$ such that $\delta q \in \mathcal{D}_{q}$ at each point of the underlying curve $q(t)$. One can of course equivalently write these equations in terms of Lagrange multipliers.

Let $\left\{\omega^{a}, a=1, \ldots, p\right\}$ be a set of $p$ independent one forms whose vanishing describes the constraints. Choose a local coordinate chart $q=(r, s) \in \mathbb{R}^{n-p} \times \mathbb{R}^{p}$, which we write as $q^{i}=\left(r^{\alpha}, s^{a}\right)$, where $1 \leqslant \alpha \leqslant n-p$ and $1 \leqslant a \leqslant p$ such that

$$
\omega^{a}(q)=\mathrm{d} s^{a}+A_{\alpha}^{a}(r, s) \mathrm{d} r^{\alpha}
$$

for all $a=1, \ldots, p$. In these coordinates, the constraints are described by vectors $v^{i}=\left(v^{\alpha}, v^{a}\right)$ satisfying $v^{a}+A_{\alpha}^{a} v^{\alpha}=0$ (a sum on repeated indices over their range is understood).

The equations of motion for the system are given by (2.1) where we choose variations $\delta q(t)$ that satisfy the constraints, i.e. $\omega^{a}(q) \delta q=0$, or equivalently, $\delta s^{a}+A_{\alpha}^{a} \delta r^{\alpha}=0$, where $\delta q^{i}=\left(\delta r^{\alpha}, \delta s^{a}\right)$. Substituting variations of this type, with $\delta r^{\alpha}$ arbitrary, into (2.1) gives

$$
\left(\frac{\mathrm{d}}{\mathrm{d} t} \frac{\partial L}{\partial \dot{r}^{\alpha}}-\frac{\partial L}{\partial r^{\alpha}}\right)=A_{\alpha}^{a}\left(\frac{\mathrm{d}}{\mathrm{d} t} \frac{\partial L}{\partial \dot{s}^{a}}-\frac{\partial L}{\partial s^{a}}\right)
$$

for all $\alpha=1, \ldots, n-p$. Equations (2.2), combined with the constraint equations

$$
\dot{s}^{a}=-A_{\alpha}^{a} \dot{r}^{\alpha}
$$

for all $a=1, \ldots, p$, give the complete equations of motion of the system.

A useful way of reformulating equations (2.2) is to define a constrained Lagrangian by substituting the constraints (2.3) into the Lagrangian:

$$
L_{c}\left(r^{\alpha}, s^{a}, \dot{r}^{\alpha}\right):=L\left(r^{\alpha}, s^{a}, \dot{r}^{\alpha},-A_{\alpha}^{a}(r, s) \dot{r}^{\alpha}\right) .
$$

The equations of motion can be written in terms of the constrained Lagrangian in the following way, as a direct coordinate calculation shows:

$$
\frac{\mathrm{d}}{\mathrm{d} t} \frac{\partial L_{c}}{\partial \dot{r}^{\alpha}}-\frac{\partial L_{c}}{\partial r^{\alpha}}+A_{\alpha}^{a} \frac{\partial L_{c}}{\partial s^{a}}=-\frac{\partial L}{\partial \dot{s}^{b}} B_{\alpha \beta}^{b} \dot{r}^{\beta},
$$

where $B_{\alpha \beta}^{b}$ is defined by

$$
B_{\alpha \beta}^{b}=\left(\frac{\partial A_{\alpha}^{b}}{\partial r^{\beta}}-\frac{\partial A_{\beta}^{b}}{\partial r^{\alpha}}+A_{\alpha}^{a} \frac{\partial A_{\beta}^{b}}{\partial s^{a}}-A_{\beta}^{a} \frac{\partial A_{\alpha}^{b}}{\partial s^{a}}\right) .
$$

Geometrically, the $A_{\alpha}^{a}$ are the coordinate expressions for the Ehresmann connection on the tangent bundle defined by the constraints, while the $B_{\alpha \beta}^{b}$ are the corresponding curvature terms (see Bloch et al (1996)).

\subsection{Symmetries}

As we shall see shortly, symmetries play an important role in our analysis. We begin here with just a few preliminary notions. Suppose we are given a nonholonomic system with Lagrangian $L: T Q \rightarrow \mathbb{R}$, and a (nonintegrable) constraint distribution $\mathcal{D}$. We can then look for a group $G$ that acts freely and properly on the configuration space $Q$. It induces an action on the tangent space $T Q$ and so it makes sense to ask that the Lagrangian $L$ be invariant. Also, one can ask 
that the distribution be invariant in the sense that the action by a group element $g \in G$ maps the distribution $\mathcal{D}_{q}$ at the point $q \in Q$ to the distribution $\mathcal{D}_{g q}$ at the point $g q$. If these properties hold, we say that $G$ is a symmetry group. The manifold $Q / G$ is called the shape space of the system and the configuration space has the structure of a principal fibre bundle $\pi: Q \rightarrow Q / G$.

\subsection{The geometry of nonholonomic systems with symmetry}

Consider a nonholonomic system with the Lagrangian $L: T Q \rightarrow \mathbb{R}$, the (nonintegrable) constraint distribution $\mathcal{D}$, and the symmetry group $G$ in the sense explained previously.

Orbits and shape space. The group orbit through a point $q$, an (immersed) submanifold, is denoted

$$
\operatorname{Orb}(q):=\{g q \mid g \in G\} .
$$

Let $\mathfrak{g}$ denote the Lie algebra of the Lie group $G$. For an element $\xi \in \mathfrak{g}$, we denote by $\xi_{Q}$ the vector field on $Q$ arising from the corresponding infinitesimal generator of the group action, so these are also the tangent spaces to the group orbits. Define, for each $q \in Q$, the vector subspace $\mathfrak{g}^{q}$ to be the set of Lie algebra elements in $\mathfrak{g}$ whose infinitesimal generators evaluated at $q$ lie in both $\mathcal{D}_{q}$ and $T_{q}(\operatorname{Orb}(q))$ :

$$
\mathfrak{g}^{q}:=\left\{\xi \in \mathfrak{g} \mid \xi_{Q}(q) \in \mathcal{D}_{q} \cap T_{q}(\operatorname{Orb}(q))\right\} .
$$

The corresponding bundle over $Q$ whose fibre at the point $q$ is given by $\mathfrak{g}^{q}$, is denoted by $\mathfrak{g}^{\mathcal{D}}$.

Reduced dynamics. Assuming that the Lagrangian and the constraint distribution are $G$-invariant, we can form the reduced velocity phase space $T Q / G$ and the reduced constraint space $\mathcal{D} / G$. The Lagrangian $L$ induces well defined functions, the reduced Lagrangian

$$
l: T Q / G \rightarrow \mathbb{R}
$$

and the constrained reduced Lagrangian

$$
l_{c}: \mathcal{D} / G \rightarrow \mathbb{R},
$$

satisfying $L=l \circ \pi_{T Q}$ and $\left.L\right|_{\mathcal{D}}=l_{c} \circ \pi_{\mathcal{D}}$ where $\pi_{T Q}: T Q \rightarrow T Q / G$ and $\pi_{\mathcal{D}}: \mathcal{D} \rightarrow \mathcal{D} / G$ are the projections. By general considerations, the Lagrange-d'Alembert equations induce well defined reduced equations on $\mathcal{D} / G$. That is, the vector field on the manifold $\mathcal{D}$ determined by the Lagrange-d'Alembert equations (including the constraints) is $G$-invariant, and so defines a reduced vector field on the quotient manifold $\mathcal{D} / G$. Following Cendra et al (2001), we call these equations the Lagrange-d'Alembert-Poincaré equations.

Let a local trivialization be chosen on the principle bundle $\pi: Q \rightarrow Q / G$, with a local representation having components denoted $(r, g)$. Let $r$, an element of the shape space $Q / G$, have coordinates denoted $r^{\alpha}$, and let $g$ be group variables for the fibre, $G$. In such a representation, the action of $G$ is the left action of $G$ on the second factor. The coordinates $(r, g)$ induce the coordinates $(r, \dot{r}, \xi)$ on $T Q / G$, where $\xi=g^{-1} \dot{g}$. The Lagrangian $L$ is invariant under the left action of $G$ and so it depends on $g$ and $\dot{g}$ only through the combination $\xi=g^{-1} \dot{g}$. Thus the reduced Lagrangian $l$ is given by

$$
l(r, \dot{r}, \xi)=L(r, g, \dot{r}, \dot{g}) .
$$

Therefore the full system of equations of motion consists of the following two groups:

(i) The Lagrange-d'Alembert-Poincaré equation on $\mathcal{D} / G$ (see theorem 2.3).

(ii) The reconstruction equation

$$
\dot{g}=g \xi .
$$


The nonholonomic momentum in the body representation. Choose a $q$-dependent basis $e_{A}(q)$ for the Lie algebra such that the first $m$ elements span the subspace $\mathfrak{g}^{q}$ in the following way. First, one chooses, for each $r$, such a basis at the identity element $g=\mathrm{Id}$, say

$$
e_{1}(r), e_{2}(r), \ldots, e_{m}(r), e_{m+1}(r), \ldots, e_{k}(r) .
$$

Now define the body fixed basis by

$$
e_{A}(r, g)=\operatorname{Ad}_{g} e_{A}(r) .
$$

Then the first $m$ elements will indeed span the subspace $\mathfrak{g}^{q}$ since the distribution is invariant. We denote the structure constants of the Lie algebra relative to this basis by $C_{A B}^{C}$.

To avoid confusion, we make the following index conventions:

(i) The first batch of indices range from 1 to $m$ corresponding to the symmetry directions along the constraint space. These indices will be denoted $a, b, c, \ldots$

(ii) The second batch of indices range from $m+1$ to $k$ corresponding to the symmetry directions not aligned with the constraints. Indices for this range will be denoted by $a^{\prime}, b^{\prime}, c^{\prime}, \ldots$

(iii) The indices $A, B, C, \ldots$ on the Lie algebra $\mathfrak{g}$ range from 1 to $k$.

(iv) The indices $\alpha, \beta, \ldots$ on the shape variables $r$ range from 1 to $\sigma$. Thus, $\sigma$ is the dimension of the shape space $Q / G$ and so $\sigma=n-k$.

The summation convention for all of these indices will be understood.

Assume that the Lagrangian has the form of kinetic minus potential energy, and that the constraints and the orbit directions span the entire tangent space to the configuration space:

$$
\mathcal{D}_{q}+T_{q}(\operatorname{Orb}(q))=T_{q} Q .
$$

Then it is possible to introduce a new Lie algebra variable $\Omega$ called the body angular velocity such that:

(i) $\Omega=\mathcal{A} \dot{r}+\xi$, where the Lie algebra valued form $\mathcal{A}=\mathcal{A}_{\alpha}^{A} e_{A}(r) \mathrm{d} r^{\alpha}$ is called the nonholonomic connection (see Bloch et al (1996) for details).

(ii) The constraints are given by $\Omega \in \operatorname{span}\left\{e_{1}(r), \ldots, e_{m}(r)\right\}$ or $\Omega^{m+1}=\cdots=\Omega^{k}=0$.

(iii) The reduced Lagrangian in the variables $(r, \dot{r}, \Omega)$ becomes

$$
l\left(r^{\alpha}, \dot{r}^{\alpha}, \Omega^{A}\right)=\frac{1}{2} g_{\alpha \beta} \dot{r}^{\alpha} \dot{r}^{\beta}+\frac{1}{2} \mathbb{I}_{A B} \Omega^{A} \Omega^{B}+\lambda_{a^{\prime} \alpha} \dot{r}^{\alpha} \Omega^{a^{\prime}}-U(r) .
$$

Here $g_{\alpha \beta}$ are coefficients of the kinetic energy metric induced on the manifold $Q / G$ and $\mathbb{I}_{A B}$ are components of the locked inertia tensor defined by

$$
\langle\mathbb{I}(r) \xi, \eta\rangle=\left\langle\left\langle\xi_{Q}, \eta_{Q}\right\rangle\right\rangle, \quad \xi, \eta \in \mathfrak{g},
$$

where $\langle\langle\cdot, \cdot\rangle\rangle$ is the kinetic energy metric. The coefficients $\lambda_{a^{\prime} \alpha}$ are defined by

$$
\lambda_{a^{\prime} \alpha}=\frac{\partial^{2} l}{\partial \xi^{a^{\prime}} \partial r^{\alpha}}-\frac{\partial^{2} l}{\partial \xi^{a^{\prime}} \partial \xi^{B}} \mathcal{A}_{\alpha}^{B} .
$$

The constrained reduced Lagrangian becomes especially simple in the variables $(r, \dot{r}, \Omega)$ :

$$
l_{c}=\frac{1}{2} g_{\alpha \beta} \dot{r}^{\alpha} \dot{r}^{\beta}+\frac{1}{2} \mathbb{I}_{a b} \Omega^{a} \Omega^{b}-U .
$$

We remark that this choice of $\Omega$ block-diagonalizes the kinetic energy metric, i.e. eliminates the terms proportional to $\Omega^{a} \dot{r}^{\alpha}$ in (2.6).

As the following proposition shows, the coefficients $\lambda_{a^{\prime} \alpha}$ in (2.5) measure the 'difference' between the nonholonomic connection and the mechanical connection for the unconstrained Lagrangian. 
Proposition 2.2. The mechanical and nonholonomic connections are identical if and only if $\lambda_{a^{\prime} \alpha}=0$.

Proof. We know that the unconstrained reduced Lagrangian is represented by (2.5) in the body angular velocity variables, which are given by

$$
\Omega=\mathcal{A} \dot{r}+\xi
$$

where $\mathcal{A}$ is the nonholonomic connection. Observe that the velocity shift associated with the nonholonomic connection eliminates the terms $\dot{r}^{\alpha} \Omega^{a}$ from the reduced Lagrangian (2.5) written in the coordinates $(r, \dot{r}, \Omega)$ on the reduced phase space. On the other hand, if the body angular velocity for the unconstrained system is introduced by

$$
\Omega=\mathcal{A}_{\text {mech }} \dot{r}+\xi
$$

where $\mathcal{A}_{\text {mech }}$ is the mechanical connection, all of the terms $\dot{r}^{\alpha} \Omega^{A}$ vanish (see Marsden (1992) for details). Therefore the two connections become identical if the terms $\dot{r}^{\alpha} \Omega^{a^{\prime}}$ vanish after the velocity shift due to the nonholonomic connection.

The nonholonomic momentum in the body representation is defined by

$$
p_{a}=\frac{\partial l}{\partial \Omega^{a}}=\frac{\partial l_{c}}{\partial \Omega^{a}}, \quad a=1, \ldots, m .
$$

Notice that the nonholonomic momentum may be viewed as a collection of components of the ordinary momentum map along the constraint directions.

The Lagrange-d'Alembert-Poincaré equations. As in Bloch et al (1996), the reduced equations of motion are given by the next theorem.

Theorem 2.3. The following reduced nonholonomic Lagrange-d'Alembert-Poincaré equations hold for each $1 \leqslant \alpha \leqslant \sigma$ and $1 \leqslant a \leqslant m$ :

$$
\begin{gathered}
\frac{\mathrm{d}}{\mathrm{d} t} \frac{\partial l_{c}}{\partial \dot{r}^{\alpha}}-\frac{\partial l_{c}}{\partial r^{\alpha}}=-\mathcal{D}_{b \alpha}^{c} I^{b d} p_{c} p_{d}-\mathcal{K}_{\alpha \beta \gamma} \dot{r}^{\beta} \dot{r}^{\gamma}-\left(\mathcal{B}_{\alpha \beta}^{c}-I_{c^{\prime} a^{\prime}} I^{a^{\prime} c} \mathcal{B}_{\alpha \beta}^{c^{\prime}}+\mathcal{D}_{b \beta \alpha} I^{b c}\right) p_{c} \dot{r}^{\beta}, \\
\dot{p}_{a}=\left(C_{b a}^{c}-C_{b a}^{c^{\prime}} I_{c^{\prime} a^{\prime}} I^{a^{\prime} c}\right) I^{b d} p_{c} p_{d}+\mathcal{D}_{a \alpha}^{c} p_{c} \dot{r}^{\alpha}+\mathcal{D}_{a \alpha \beta} \dot{r}^{\alpha} \dot{r}^{\beta} .
\end{gathered}
$$

Here and below, $l_{c}\left(r^{\alpha}, \dot{r}^{\alpha}, \Omega^{a}\right)$ is the constrained Lagrangian, and $I^{b d}$ and $I_{a^{\prime} c^{\prime}}$ are the inverse of the tensors $\left.\mathbb{I}\right|_{\mathfrak{g}^{q}}$ and $\left.\mathbb{I}^{-1}\right|_{\left(\mathfrak{g}^{q}\right)^{*}}$, respectively. We stress that in general $I^{b d} \neq \mathbb{I}^{b d}$ and $I_{a^{\prime} c^{\prime}} \neq \mathbb{I}_{a^{\prime} c^{\prime}}$. The coefficients $\mathcal{B}_{\alpha \beta}^{C}, \mathcal{D}_{b \alpha}^{c}, \mathcal{D}_{b \alpha \beta}, \mathcal{K}_{\alpha \beta \gamma}$ are given by the formulae

$$
\begin{gathered}
\mathcal{D}_{b \alpha}^{c}=-\left(C_{A b}^{c}-C_{A b}^{c^{\prime}} I_{c^{\prime} a^{\prime}} I^{a^{\prime} c}\right) \mathcal{A}_{\alpha}^{A}+C_{a b}^{c^{\prime}} \lambda_{c^{\prime} \alpha} I^{a c}+\gamma_{b \alpha}^{c}-\gamma_{b \alpha}^{c^{\prime}} I_{c^{\prime} a^{\prime}} I^{a^{\prime} c}, \\
\mathcal{B}_{\alpha \beta}^{C}=\frac{\partial \mathcal{A}_{\alpha}^{C}}{\partial r^{\beta}}-\frac{\partial \mathcal{A}_{\beta}^{C}}{\partial r^{\alpha}}-C_{B A}^{C} \mathcal{A}_{\alpha}^{A} \mathcal{A}_{\beta}^{B}+\gamma_{A \beta}^{C} \mathcal{A}_{\alpha}^{A}-\gamma_{A \alpha}^{C} \mathcal{A}_{\beta}^{A}, \\
\mathcal{D}_{b \alpha \beta}=\lambda_{c^{\prime} \beta}\left(\gamma_{b \alpha}^{c^{\prime}}-C_{A b}^{c^{\prime}} \mathcal{A}_{\alpha}^{A}\right), \\
\mathcal{K}_{\alpha \beta \gamma}=\lambda_{c^{\prime} \gamma} \mathcal{B}_{\alpha \beta}^{c^{\prime}}
\end{gathered}
$$

and the coefficients $\gamma_{B \alpha}^{C}$ are defined by

$$
\frac{\partial e_{B}}{\partial r^{\alpha}}=\gamma_{B \alpha}^{C} e_{C}
$$

Equations (2.7) and (2.8) generalize the equations of motion in the orthogonal body frame (see Bloch et al (1996)). Here we no longer assume that the body frame is orthogonal. The 
derivation of these equations follows the procedure of obtaining equations (2.4) outlined earlier in this section.

\section{Invariant measures of the Euler-Poincaré-Suslov equations}

An important special case of the reduced nonholonomic equations is the case when there is no shape space at all. In this case the system is characterized by the Lagrangian $L=\frac{1}{2} \mathbb{I}_{A B} \Omega^{A} \Omega^{B}$ and the left-invariant constraint

$$
\langle a, \Omega\rangle=a_{A} \Omega^{A}=0 \text {. }
$$

Here $a=a_{A} e^{A} \in \mathfrak{g}^{*}$ and $\Omega=\Omega^{A} e_{A}$, where $e_{A}, A=1, \ldots, k$, is a basis for $\mathfrak{g}$ and $e^{A}$ is its dual basis. Multiple constraints may be imposed as well. The two classical examples of such systems are the Chaplygin sleigh and the Suslov problem. These problems were introduced by Chaplygin in 1895 and Suslov in 1902, respectively.

We can consider the problem of when such systems exhibit asymptotic behaviour. Following Kozlov (1988) it is convenient to consider the unconstrained case first. In the absence of constraints the dynamics is governed by the basic Euler-Poincaré equations

$$
\dot{p}_{B}=C_{A B}^{C} \mathbb{I}^{A D} p_{C} p_{D}=C_{A B}^{C} p_{C} \Omega^{A},
$$

where $p_{B}=\mathbb{I}_{A B} \Omega^{B}$ are the components of the momentum $p \in \mathfrak{g}^{*}$. One considers the question of whether the (unconstrained) equations (3.2) have an absolutely continuous integral invariant $f d^{k} \Omega$ with summable density $\mathcal{M}$. If $\mathcal{M}$ is a positive function of class $C^{1}$ one calls the integral invariant an invariant measure. Kozlov (1988) shows the following theorem.

Theorem 3.1. The Euler-Poincaré equations have an invariant measure if and only if the group $G$ is unimodular.

Neither direction is hard to prove but we content ourselves with proving sufficiency here.

A group is said to be unimodular if it has a bilaterally invariant measure. A criterion for unimodularity is $C_{A C}^{C}=0$ (using the Einstein summation convention). Now we know (Liouville's theorem) that the flow of a vector differential equation $\dot{x}=f(x)$ is phase volume preserving if and only if $\operatorname{div} f=0$. In this case the divergence of the right-hand side of equation (3.2) is $C_{A C}^{C} \mathbb{I}^{A D} p_{D}=0$. The statement of the theorem now follows from the following theorem of Kozlov (1998): A flow due to a homogeneous vector field in $\mathbb{R}^{n}$ is measure-preserving if and only if this flow preserves the standard volume in $\mathbb{R}^{n}$.

Now, turning to the case where we have the constraint (3.1) we obtain the Euler-PoincaréSuslov equations

$$
\dot{p}_{B}=C_{A B}^{C} \mathbb{I}^{A D} p_{C} p_{D}+\lambda a_{B}=C_{A B}^{C} p_{C} \Omega^{A}+\lambda a_{B}
$$

together with the constraint (3.1). Here $\lambda$ is the Lagrange multiplier. This defines a system on the subspace of the dual Lie algebra defined by the constraint. Since the constraint is assumed to be nonholonomic, this subspace is not a subalgebra. One can then formulate a condition for the existence of an invariant measure of the Euler-Poincaré-Suslov equations.

Theorem 3.2. Equations (3.3) have an invariant measure if and only if

$$
K \operatorname{ad}_{\mathbb{I}^{-1} a}^{*} a+T=\mu a, \quad \mu \in \mathbb{R},
$$

where $K=1 /\left\langle a, \mathbb{I}^{-1} a\right\rangle$ and $T \in \mathfrak{g}^{*}$ is defined by $\langle T, \xi\rangle=\operatorname{Tr}\left(\operatorname{ad}_{\xi}\right)$. 
This theorem was proved by Kozlov (1988) for compact algebras and for arbitrary algebras by Jovanović (1998). In coordinates, condition (3.4) becomes

$$
K C_{A B}^{C} \mathbb{I}^{A D} a_{C} a_{D}+C_{B C}^{C}=\mu a_{B} .
$$

For a compact algebra (3.4) becomes

$$
\left[\mathbb{I}^{-1} a, a\right]=\mu a, \quad \mu \in \mathbb{R},
$$

where we identified $\mathfrak{g}^{*}$ with $\mathfrak{g}$.

The proof of theorem 3.2 reduces to the computation of the divergence of the vector field in (3.3). To accomplish this calculation, one first chooses a basis of $\mathfrak{g}$ such that $a_{1}=\cdots=a_{n-1}=0$ and $a_{n}=1$. Then the first $n-1$ equations of (3.3) are a closed system of Euler-Poincaré equations on the hyperplane $\Omega_{n}=0$ which is invariant under the flow. Computing the divergence of the corresponding vector field, one concludes that this divergence vanishes if and only if the condition (3.4) is satisfied. The flow then preserves the standard measure. The final equation plus the constraint determines the Lagrange multiplier $\lambda$. One can introduce several constraints of this type (see Kozlov (1988)).

In the compact case only constraint vectors $a$ which commute with $\mathbb{I}^{-1} a$ allow the measure to be preserved. This means that $a$ and $\mathbb{I}^{-1} a$ must lie in the same maximal commuting subalgebra. In particular, if $a$ is an eigenstate of the inertia tensor, the reduced phase volume is preserved. When the maximal commuting subalgebra is one-dimensional this is a necessary condition. This is the case for groups such as $S O(3)$.

We thus have the following result which reflects a symmetry requirement on the constraints.

Theorem 3.3. A compact Euler-Poincaré-Suslov system is measure preserving (i.e. does not exhibit asymptotic dynamics) if the constraint vectors a are eigenvectors of the inertia tensor, or if the constrained system is $\mathbb{Z}_{2}$ symmetric about each of its principal axes. If the maximal commuting subalgebra is one-dimensional this condition is necessary.

\section{Invariant measures of systems with internal degrees of freedom}

In this section we extend the result of Kozlov (1988) and Jovanović (1998) to nonholonomic systems with nontrivial shape space. One can think of these systems as the Euler-PoincaréSuslov systems with internal degrees of freedom. Recall that the constraints are of the form $\Omega^{m+1}=\cdots=\Omega^{k}=0$. To simplify the exposition, we consider below systems with a single constraint. The results are valid for systems with multiple constraints as well.

Consider a nonholonomic system with reduced Lagrangian $l(r, \dot{r}, \Omega)$ and a constraint $\langle a(r), \Omega\rangle=0$. The subspace of the Lie algebra defined by the constraint at the configuration $q$ is denoted here by $\mathfrak{g}^{q}$. The orientation of this subspace in $\mathfrak{g}$ depends on the shape configuration of the system, $r$. The dimension of $\mathfrak{g}^{q}$ however stays the same. As discussed in section 2, we choose a special moving frame in which $\mathfrak{g}^{q}$ is spanned by the vectors $e_{1}(r), \ldots, e_{k-1}(r)$. The equation of the constraint in this basis becomes $\Omega^{k}=0$. Recall that the horizontal part of the kinetic energy metric is $g_{\alpha \beta}(r)$.

Theorem 4.1. The system associated with the reduced Lagrangian $l(r, \dot{r}, \Omega)$ and the constraint $\langle a(r), \Omega\rangle=0$ has an integral invariant with a $C^{1}$ density $\mathcal{M}(r)$ if and only if

(i) $\left(C_{b a}^{a}-C_{b a}^{k} \frac{\mathbb{I}^{k a}}{\mathbb{I}^{k k}}\right)-g^{\alpha \delta} \mathcal{D}_{b \alpha \delta}=0$.

(ii) The form $\left(\mathcal{D}_{b \beta}^{b}-g^{\alpha \delta} \lambda_{k \delta} \mathcal{B}_{\alpha \beta}^{k}\right) \mathrm{d} r^{\beta}$ is exact. 
Proof. Recall that the reduced equations of motion are (2.7) and (2.8). We now rewrite the shape equation exposing more details. The shape equations (2.7) become

$$
\begin{gathered}
g_{\alpha \beta} \ddot{r}^{\beta}+\dot{g}_{\alpha \beta} \dot{r}^{\alpha} \dot{r}^{\beta}-\frac{1}{2} \frac{\partial g_{\beta \gamma}}{\partial r^{\alpha}} \dot{r}^{\beta} \dot{r}^{\gamma}=-\frac{1}{2} \frac{\partial I^{a b}}{\partial r^{\alpha}} p_{a} p_{b}-\frac{\partial U}{\partial r^{\alpha}}-\mathcal{D}_{b \alpha}^{c} I^{b d} p_{c} p_{d} \\
-\left(\mathcal{B}_{\alpha \beta}^{c}-\frac{\mathbb{I}^{k c}}{\mathbb{I}^{k k}} \mathcal{B}_{\alpha \beta}^{k}+\mathcal{D}_{b \alpha \beta} I^{b c}\right) p_{c} \dot{r}^{\beta}-\mathcal{K}_{\alpha \beta \gamma} \dot{r}^{\beta} \dot{r}^{\gamma}
\end{gathered}
$$

Solving these equations for $\ddot{r}^{\beta}$ we obtain

$$
\begin{aligned}
\ddot{r}^{\delta}= & g^{\delta \alpha}\left(\frac{1}{2} \frac{\partial g_{\beta \gamma}}{\partial r^{\alpha}}-\frac{\partial g_{\alpha \beta}}{\partial r^{\alpha}}\right) \dot{r}^{\beta} \dot{r}^{\gamma}-g^{\alpha \delta}\left(\frac{1}{2} \frac{\partial I^{b c}}{\partial r^{\alpha}}-g^{\alpha \delta} \mathcal{D}_{b \alpha}^{c} I^{b d}\right) p_{c} p_{d} \\
& -g^{\alpha \delta} \frac{\partial U}{\partial r^{\alpha}}-g^{\alpha \delta}\left(\mathcal{B}_{\alpha \beta}^{c}-\frac{\mathbb{I}^{k c}}{\mathbb{I}^{k k}} \mathcal{B}_{\alpha \beta}^{k}+\mathcal{D}_{b \alpha \beta} I^{b c}\right) p_{c} \dot{r}^{\beta}-g^{\alpha \delta} \mathcal{K}_{\alpha \beta \gamma} \dot{r}^{\beta} \dot{r}^{\gamma} .
\end{aligned}
$$

These equations should be of course coupled with the momentum equation.

According to Liouville's theorem, the equation for the density $\mathcal{M}(r)$ of the invariant measure is $\operatorname{div}(\mathcal{M} F)=0$ where $F$ is the vector field determined by the equations of motion. For an $\mathcal{M}$ that depends on the shape configuration variables only, this equation becomes

$$
\begin{aligned}
\frac{\partial \mathcal{M}}{\partial r^{\beta}} \dot{r}^{\beta}+\mathcal{M} g^{\alpha \delta} & \frac{\partial}{\partial \dot{r}^{\delta}}\left[\left(\frac{1}{2} \frac{\partial g_{\beta \gamma}}{\partial r^{\alpha}}-\frac{\partial g_{\alpha \beta}}{\partial r^{\alpha}}\right) \dot{r}^{\beta} \dot{r}^{\gamma}\right. \\
& \left.-\mathcal{K}_{\alpha \beta \gamma} \dot{r}^{\beta} \dot{r}^{\gamma}-\left(\mathcal{B}_{\alpha \beta}^{c}-\frac{\mathbb{I}^{k c}}{\mathbb{I}^{k k}} \mathcal{B}_{\alpha \beta}^{k}+\mathcal{D}_{b \alpha \beta} I^{b c}\right) p_{c} \dot{r}^{\beta}\right] \\
& +\mathcal{M} \frac{\partial}{\partial p_{a}}\left[\left(C_{b a}^{c}-C_{b a}^{k} \frac{\mathbb{I}^{k c}}{\mathbb{I}^{k k}}\right) I^{b d} p_{c} p_{d}+\mathcal{D}_{b \alpha}^{c} p_{c} \dot{r}^{\alpha}\right]=0 .
\end{aligned}
$$

The terms linear in $p$ in this equation vanish if and only if condition (i) of the theorem is satisfied. The equation for the density $\mathcal{M}$ of the invariant measure therefore becomes

$$
\frac{1}{\mathcal{M}} \frac{\partial \mathcal{M}}{\partial r^{\beta}} \dot{r}^{\beta}=\left(g^{\alpha \delta} \frac{\partial g_{\alpha \delta}}{\partial r^{\beta}}+\mathcal{D}_{b \beta}^{b}-g^{\alpha \delta} \mathcal{K}_{\alpha \delta \beta}-g^{\alpha \delta} \mathcal{K}_{\alpha \beta \delta}\right) \dot{r}^{\beta} .
$$

Using the explicit representation for $\mathcal{K}_{\alpha \beta \gamma}$, and noticing that

$$
g^{\alpha \delta} \frac{\partial g_{\alpha \delta}}{\partial r^{\beta}} \mathrm{d} \dot{r}^{\beta}=\mathrm{d} \ln (\operatorname{det} g),
$$

we can rewrite this condition as

$$
\mathrm{d} \ln (\mathcal{M})=\mathrm{d} \ln (\operatorname{det} g)+\left(\mathcal{D}_{b \beta}^{b}-g^{\alpha \delta} \lambda_{k \delta} \mathcal{B}_{\alpha \beta}^{k}\right) \mathrm{d} r^{\beta} .
$$

We remark that the form $\mathrm{d} \ln (\operatorname{det} g$ ) is the Lagrangian representation of the phase volume form for the geodesic flow on the shape space due to the shape metric. Since the first term in the right-hand side of (4.1) is exact, the invariant measure with density depending on the shape configuration only exists if and only if condition (ii) of the theorem is satisfied. If the shape space is simply connected, this condition is equivalent to

$$
\mathrm{d}\left[\left(\mathcal{D}_{b \beta}^{b}-g^{\alpha \delta} \lambda_{k \delta} \mathcal{B}_{\alpha \beta}^{k}\right) \mathrm{d} r^{\beta}\right]=0 .
$$

Corollary 4.2. The density $\mathcal{M}$ is defined uniquely up to a constant factor.

Corollary 4.3. A system that satisfies the conditions of the theorem retains the invariant measure with the same density if an arbitrary symmetry preserving potential is added to the Lagrangian. 
Proof. Conditions (i) and (ii) of theorem 4.1 do not impose restrictions on the potential. Adding a symmetry preserving potential to the Lagrangian does not change the symmetry group and the kinetic energy metric and therefore keeps (i) and (ii) satisfied. The density of the invariant measure remains unchanged because equation (4.1) is not affected by the change in the potential energy.

We now discuss a few situations in which conditions (i) and (ii) of theorem 4.1 are satisfied. We start from condition (i) of the theorem. Taking into account the formulae for $\mathcal{D}_{a \alpha \beta}$ and $\lambda_{k \beta}$ we conclude that (i) is a restriction on the 'direction' of the constraint subspace 'relative' to the kinetic energy metric, as it is in the case of systems with trivial shape space. This condition becomes especially simple if $g^{\alpha \beta} \mathcal{D}_{a \alpha \beta}$ vanishes, which is equivalent to the skew symmetry of $\mathcal{D}_{a \alpha \beta}$ with respect to $\alpha$ and $\beta$. The latter is equivalent to the absence of the terms quadratic in $\dot{r}$ in the momentum equation. Recall that generically the relative equilibria demonstrate asymptotic behaviour if these terms are present in the momentum equation (see Zenkov et al (1998) for details). If $\mathcal{D}_{a \alpha \beta}$ are skew, condition (i) can be rewritten, in invariant form, as

$$
K(r) \operatorname{ad}_{\mathbb{I}^{-1}(r) a(r)}^{*} a(r)+T(r)=\mu(r) a(r), \quad \mu: Q / G \rightarrow \mathbb{R},
$$

which is just a 'shape-dependent' variant of (3.4). In this equation, $K(r)$ and $T(r)$ are defined by

$$
K(r)=\frac{1}{\left\langle a(r), \mathbb{I}^{-1}(r) a(r)\right\rangle}, \quad\langle T(r), \xi\rangle=\operatorname{Tr}\left(\operatorname{ad}_{\xi}\right),
$$

and we do not assume a special choice of the body frame. Recall that this special choice results in the constraint being represented by $\Omega^{k}=0$ instead of the general constraint equation $\langle a(r), \Omega\rangle=0$.

Assume now that the nonholonomic connection equals the mechanical connection. By proposition 2.2, $\lambda_{k \beta}=0$ and hence $\mathcal{D}_{a \alpha \beta}$ vanish. Condition (i) of theorem (4.1) becomes

$$
C_{b A}^{A}+C_{A b}^{k} \frac{\mathbb{I}^{k A}}{\mathbb{I}^{k k}}=0
$$

which is of course equivalent to (4.3).

Condition (ii) of the theorem now requires that the form

$$
\left[-\left(C_{B a}^{a}-C_{B a}^{k} \frac{\mathbb{I}^{k a}}{\mathbb{I}^{k k}}\right) \mathcal{A}_{\beta}^{B}+\gamma_{a \beta}^{a}-\gamma_{a \beta}^{k} \frac{\mathbb{I}^{k a}}{\mathbb{I}^{k k}}\right] \mathrm{d} r^{\beta}
$$

is exact. Using (4.4), we rewrite the form in condition (ii) as

$$
\left[-\left(C_{k A}^{A}-C_{k A}^{k} \frac{\mathbb{I}^{k A}}{\mathbb{I}^{k k}}\right) \mathcal{A}_{\beta}^{k}+\gamma_{A \beta}^{A}-\gamma_{A \beta}^{k} \frac{\mathbb{I}^{k A}}{\mathbb{I}^{k k}}\right] \mathrm{d} r^{\beta} .
$$

Observe that the origins of the quantities $C_{A B}^{C}$ and $\gamma_{B \beta}^{A}$ are the same. They are the coefficients of the expansion of the Jacobi-Lie bracket of the vector fields $\partial_{\alpha}$ and $e_{A}(r, g)$. Recall that we use these fields as the basis for expansion of $\dot{q}$, the velocity of the system.

Assume now that the group $G$ is unimodular (we keep the requirement $\lambda_{k \beta}=0$ ). Then, since $C_{B A}^{A}=0,(4.4)$ is equivalent to $C_{A B}^{k} \mathbb{I}^{k A}=0$. Using invariant notations, we can rewrite this condition as $\operatorname{ad}_{\mathbb{I}^{-1} a}^{*} a=0$. Hence (4.5) becomes

$$
\left(\gamma_{A \beta}^{A}-\gamma_{A \beta}^{k} \frac{\mathbb{I}^{k A}}{\mathbb{I}^{k k}}\right) \mathrm{d} r^{\beta}
$$

The basis $e_{A}(r)$ of the Lie algebra $\mathfrak{g}$ (which we treat as a linear space for now) can be viewed as a transformation of some fixed basis $e_{A}$ :

$$
e_{A}(r)=R_{A}^{B}(r) e_{B} .
$$


From the definition of $\gamma_{A \alpha}^{B}$ we obtain:

$$
\frac{\partial e_{A}(r)}{\partial r^{\alpha}}=\frac{\partial R_{A}^{B}}{\partial r^{\alpha}} e_{B}=\gamma_{A \alpha}^{C} R_{C}^{B} e_{B},
$$

which implies

$$
\gamma_{A \alpha}^{C}=\left(R^{-1} \partial_{\alpha} R\right)_{A}^{C}
$$

If the matrix $R(r)$ can be chosen to be orthogonal for each $r$, then $R^{-1} R$ is skew, and thus $\gamma_{A \alpha}^{A}=0$. In this situation form (4.6) becomes

$$
-\gamma_{A \beta}^{k} \frac{\mathbb{I}^{k A}}{\mathbb{I}^{k k}} \mathrm{~d} r^{\beta}
$$

This differential form simplifies if, for example, the body frame $e_{A}(r)$ was chosen orthogonal with respect to the kinetic energy metric. In this case (4.7) becomes $-\gamma_{k \beta}^{k} \mathrm{~d} r^{\beta}$. The latter is closed if the vector $e_{k}(r)$ is independent of all shape variables but one. One can of course extend the list of simple cases of when condition (ii) of theorem 4.1 is satisfied. Below we discuss and illustrate two important cases.

Systems with one-dimensional shape space. Assume that condition (i) of theorem 4.1 is satisfied. In this case the equation for the density of the invariant measure becomes

$$
\mathrm{d}(\ln \mathcal{M})=\mathrm{d}(\ln g)+\mathcal{D}_{b}^{b} \mathrm{~d} r .
$$

The solution of this equation is globally defined if the shape space is either noncompact (and thus diffeomorphic to $\mathbb{R}$ ), or compact and the average of the function $\mathcal{D}_{b}^{b}$ equals zero.

Systems with conserved momentum. If the nonholonomic momentum is a constant of motion, then condition (i) of theorem 4.1 is trivially satisfied. Moreover, condition (ii) now asks that the form

$$
g^{\alpha \delta} \lambda_{k \delta} \mathcal{B}_{\alpha \beta}^{k} \mathrm{~d} r^{\beta}
$$

is exact. The system thus preserves the measure with the density

$$
\mathcal{M}=\operatorname{det} g \exp \left(-\int g^{\alpha \delta} \lambda_{k \delta} \mathcal{B}_{\alpha \beta}^{k} \mathrm{~d} r^{\beta}\right) .
$$

\section{Examples}

Here we consider examples of nonholonomic systems that have an invariant measure and show how this measure can be found using our theory.

The Routh problem. This mechanical system consists of a uniform sphere rolling without slipping on the inner surface of a vertically oriented surface of revolution. Apparently Routh was the first to explore this problem. He described the family of stationary periodic motions and obtained a necessary condition for stability of these motions. Routh noticed as well that integration of the equations of motion may be reduced to integration of a system of two linear differential equations with variable coefficients and considered a few cases when the equations of motion can be solved by quadratures. Modern references that treat this system are Hermans (1995) and Zenkov (1995).

This problem is $S O(2) \times S O(2)$-invariant, where the first copy of $S O(2)$ represents rotations about the axis of the surface of revolution while the second copy of $S O(2)$ represents rotations of the sphere about its radius through the contact point of the surface and the sphere. 
Let $r$ be the latitude of this contact point, $a$ be the radius of the sphere, $c(r)+a$ be the reciprocal of the curvature of the meridian of the surface, and $b(r)$ be the distance from the axis of the surface to the sphere's centre. The shape metric is $c^{2}(r) \dot{r}^{2} / 2$ while the momentum equations are

$$
\dot{p}_{1}=\frac{c(r) \sin r}{b(r)} p_{1} \dot{r}-\frac{2}{7} p_{2} \dot{r}, \quad \dot{p}_{2}=\left(1-\frac{c(r) \cos r}{b(r)}\right) p_{1} \dot{r} .
$$

See Zenkov (1995) for details and in particular for the choice of the Lie algebra basis and a physical interpretation of the components of nonholonomic momentum.

The shape space is one-dimensional, the symmetry group $S O(2) \times S O(2)$ is commutative, and there are no terms proportional to $\dot{r}^{2}$ in the momentum equations. The trace term in (4.8) equals $c(r) \sin r / b(r)$, and thus the density of the invariant measure for the Routh problem is

$$
\mathcal{M}=c^{2}(r) \exp \left(\int \frac{c(r) \sin r}{b(r)} \mathrm{d} r\right) .
$$

The group action in this problem is singular: the intersection points of the surface of revolution and its axis have nontrivial isotropy subgroups. The shape coordinate $r$ equals $\pm \pi / 2$ at these points. As a result,

$$
\lim _{r \rightarrow-\pi / 2} \mathcal{M}(r)=\lim _{r \rightarrow \pi / 2} \mathcal{M}(r)=\infty .
$$

The falling disc. Consider a homogeneous disc rolling without sliding on a horizontal plane. This mechanical system is $S O(2) \times S E(2)$-invariant; the group $S O(2)$ represents the symmetry of the disc while the group $S E(2)$ represents the Euclidean symmetry of the overall system.

Classical references for the rolling disc are Vierkandt (1892), Korteweg (1899), and Appel (1900). In particular, Vierkandt showed that on the reduced space $\mathcal{D} / S E(2)$ - the constrained velocity phase space modulo the action of the Euclidean group $S E(2)$-most orbits of the system are periodic.

The shape of the system is specified by a single coordinate- the tilt of the disc denoted here by $r$. The momentum equations are

$$
\begin{aligned}
& \dot{p}_{1}=m R^{2}\left(-\frac{\sin r}{A \cos r} p_{1}+\left(\frac{\cos r}{m R^{2}+B}+\frac{\sin ^{2} r}{A \cos r}\right) p_{2}\right) \dot{r} \\
& \dot{p}_{2}=m R^{2}\left(-\frac{1}{A \cos r} p_{1}+\frac{\sin r}{A \cos r} p_{2}\right) \dot{r} .
\end{aligned}
$$

Hence, the trace terms $\mathcal{D}_{b}^{b}$ in (4.8) vanish, and the density of the invariant measure equals the component of the shape metric $g(r)$. The latter equals the moment of inertia of the disc with respect to the line through the rim of the disc and parallel to its diameter. Since the density of the measure is determined up to a constant factor, we conclude that the dynamics preserves the reduced phase space volume.

The three-dimensional Chaplygin sleigh with an oscillating mass. The three-dimensional Chaplygin sleigh is a free rigid body subject to the nonholonomic constraint $v^{3}=0$, where $v^{3}$ is the third component of the (linear) velocity relative to the body frame. The Lagrangian of this system is

$$
\frac{1}{2} M\left(\left(v^{1}\right)^{2}+\left(v^{2}\right)^{2}+\left(v^{3}\right)^{2}\right)+\frac{1}{2}\left(I_{1}\left(\Omega^{1}\right)^{2}+I_{2}\left(\Omega^{2}\right)^{2}+I_{3}\left(\Omega^{3}\right)^{2}\right) .
$$

In this formula $M$ is the mass of the body, $I_{j}$ are the eigenvalues of its inertia tensor, and $\left(\Omega^{1}, \Omega^{2}, \Omega^{3}\right)$ and $\left(v^{1}, v^{2}, v^{3}\right)$ are the angular and linear velocities relative to the body frame. 
The dynamics of this system is discussed in Neimark and Fufaev (1972). Rand and Ramani (2000) point out that a constraint like this has been used to model fins on an underwater missile.

We couple this system with an oscillator moving along the third coordinate axis of the body frame. The mass of this oscillator is $m$ and the displacement from the origin is $r$. To keep the notation uniform with the general theory, we write the components of the linear velocity relative to the body frame as $\left(\Omega^{4}, \Omega^{5}, \Omega^{6}\right)$. The vector $\left(\Omega^{1}, \Omega^{2}, \Omega^{3}, \Omega^{4}, \Omega^{5}, \Omega^{6}\right)$ should be viewed as an element of the Lie algebra se(3). The Lagrangian of this new system is

$$
\begin{aligned}
L= & \frac{1}{2}\left(I_{1}\left(\Omega^{1}\right)^{2}+I_{2}\left(\Omega^{2}\right)^{2}+I_{3}\left(\Omega^{3}\right)^{2}\right)+\frac{M}{2}\left(\left(\Omega^{4}\right)^{2}+\left(\Omega^{5}\right)^{2}+\left(\Omega^{6}\right)^{2}\right) \\
& +\frac{m}{2}\left(\left(\Omega^{4}+\Omega^{2} r\right)^{2}+\left(\Omega^{5}-\Omega^{1} r\right)^{2}+\left(\Omega^{6}+\dot{r}\right)^{2}\right)-U(r) .
\end{aligned}
$$

The configuration space is $\mathbb{R} \times S E(3)$, and the system is invariant under the left action of $S E$ (3) on the second factor. We have not specified the potential energy as its choice does not affect the existence of the invariant measure (see corollary 4.3). The shape space is just the first factor of $\mathbb{R} \times S E(3)$ and is one-dimensional, and thus the above theory is applicable. To show the existence of the invariant measure, we note the following:

(i) The constrained Lagrangian does not contain terms that simultaneously depend on $\dot{r}$ and $p_{a}$. The constraint is $\Omega^{6}=0$. Therefore, all the coefficients of the nonholonomic connection as well as its curvature form vanish. This implies that the terms $\mathcal{D}_{a \alpha \beta}$ and $\mathcal{K}_{\alpha \beta \gamma}$ vanish too. The differential form from condition (ii) of theorem 4.1 is therefore trivial.

(ii) The moving frame is $r$-independent. Therefore all of the coefficients $\gamma_{A \alpha}^{B}$ are trivial. Condition (i) of theorem 4.1 is satisfied since the group $S E(3)$ is unimodular and $e_{6}$ is the eigenvector of the inertia tensor.

(iii) The shape metric is $r$-independent.

The discussion at the end of section 4 implies that the system's dynamics preserves the volume in the reduced phase space.

This can be verified by a straightforward computation of the divergence of the vector field that defines the equations of motion:

$$
\begin{aligned}
& \ddot{r}=-\frac{\partial U_{a}}{\partial r}, \\
& \dot{p}_{1}=-\Omega^{2} p_{3}+\Omega^{3} p_{2}-m \Omega^{5} \dot{r}, \\
& \dot{p}_{2}=-\Omega^{3} p_{1}+\Omega^{1} p_{3}+m \Omega^{4} \dot{r}, \\
& \dot{p}_{3}=-\Omega^{1} p_{2}+\Omega^{2} p_{1}-\Omega^{4} p_{5}+\Omega^{5} p_{4}, \\
& \dot{p}_{4}=\Omega^{3} p_{5}-m \Omega^{2} \dot{r}, \\
& \dot{p}_{5}=-\Omega^{3} p_{4}+m \Omega^{1} \dot{r} .
\end{aligned}
$$

Chaplygin sphere. This system consists of a sphere rolling without slipping on a horizontal plane. The centre of mass of this sphere is at the geometric centre, but the principal moments of inertia are distinct. Chaplygin (1903) proved integrability of this problem. Modern references for the Chaplygin sphere include Kozlov (1985) and Schneider (2002).

One may view this system as a nonholonomic version of the Euler top. The configuration space is diffeomorphic to $S O(3) \times \mathbb{R}^{2}$. We choose the Euler angles $(\theta, \phi, \psi)$ and the Cartesian 
coordinates $(x, y)$ as the configuration parameters of the Chaplygin sphere. The Lagrangian and constraints written in these coordinates become

$$
\begin{aligned}
L= & \frac{I_{1}}{2}(\dot{\theta} \cos \psi+\dot{\phi} \sin \psi \sin \theta)^{2}+\frac{I_{2}}{2}(-\dot{\theta} \sin \psi+\dot{\phi} \cos \psi \sin \theta)^{2} \\
& +\frac{I_{3}}{2}(\dot{\psi}+\dot{\phi} \cos \theta)^{2}+\frac{M}{2}\left(\dot{x}^{2}+\dot{y}^{2}\right)
\end{aligned}
$$

and

$$
\dot{x}-\dot{\theta} \sin \phi+\dot{\psi} \cos \phi \sin \theta=0, \quad \dot{y}+\dot{\theta} \cos \phi+\dot{\psi} \sin \phi \sin \theta=0,
$$

respectively.

This system is $S E(2)$-invariant. The action by the group element $(\alpha, a, b)$ on the configuration space is given by

$(\theta, \psi, \phi, x, y) \mapsto(\theta, \psi, \phi+\alpha, x \cos \alpha-y \sin \alpha+a, x \sin \alpha+y \cos \alpha+b)$.

The shape space for the Chaplygin sphere is diffeomorphic to the two-dimensional sphere. The nonholonomic momentum map has just one component and is moreover preserved. Straightforward computations show that the form (4.9) is exact. The conditions for measure existence are therefore satisfied. The density of the invariant measure is computed in overdetermined coordinates in Chaplygin (1903) (see also Kozlov (1985)).

The invariant manifolds of the Chaplygin sphere are two-dimensional tori. The phase flow on these tori is measure preserving and thus there are angle variables $(x, y)$ on each torus in which the flow equations become

$$
\dot{x}=\frac{\lambda}{\mathcal{M}(x, y)}, \quad \dot{y}=\frac{\mu}{\mathcal{M}(x, y)} .
$$

(see Kolmogorov (1953) and Kozlov (1985) for details). In general, these equations cannot be rewritten as

$$
\dot{x}=\lambda, \quad \dot{y}=\mu .
$$

The flow however becomes quasi-periodic after a time substitution $\mathrm{d} t=\mathcal{M}(x, y) \mathrm{d} \tau$ (see Kozlov (1985) for details). This example thus shows that the flow on the nonholonomic invariant tori can be more complicated than a Hamiltonian flow.

It follows from corollary 4.3 that adding a symmetry preserving potential to the Lagrangian of the Chaplygin sphere leaves the new system measure preserving with the same measure density. This was pointed out by Kozlov for a specific potential (see Kozlov (1985) for details).

\section{Conclusions}

We have given a method for analysing measure preservation in nonholonomic mechanics. We have obtained a constructive approach for computing densities of such measures in the presence of symmetry. As the examples demonstrate, the density of an invariant measure often equals $\operatorname{det} g$, where $g$ is the shape kinetic energy metric. This happens because of the special choice of the basis in the constrained subspace of the Lie algebra of the symmetry group. We intend to study this property in a forthcoming publication.

\section{Acknowledgments}

We would like to thank Professors C Doering, J Hamberg, and J Marsden for helpful discussions. DVZ's research was partially supported by a University of Michigan Rackham 
Fellowship and an NSF group infrastructure grant at the University of Michigan; AMB's research was partially supported by NSF grants DMS-9803181 and DMS-0103895, and an NSF group infrastructure grant at the University of Michigan.

\section{References}

Appel P 1900 Sur l'intégration des équations du mouvement d'un corps pesant de révolution roulant par une arête circulaire sur un plan horizontal; cas parficulier du cerceau Rendiconti del circolo matematico di Palermo 14 1-6

Arnold V I 1988 Dynamical Systems III (New York: Springer)

Blackall C J 1941 On volume integral invariant of non-holonomic dynamical systems Am. J. Math. 63 155-68

Bloch A M, Krishnaprasad P S, Marsden J E and Murray R 1996 Nonholonomic mechanical systems with symmetry Arch. Rat. Mech. Anal. 136 21-99

Cendra H, Marsden J E and Ratiu T S 2001 Lagrangian reduction by stages Mem. Am. Math. Soc. 152

Chaplygin S A 1903 On the rolling of a sphere on a horizontal plane Mat. Sbornik XXIV 139-68 (in Russian)

Jovanović B 1998 Nonholonomic geodesic flows on Lie groups and the integrable Suslov problem on SO(4) J. Phys. A: Math. Gen. 31 1415-22

Hermans J 1995 A symmetric sphere rolling on a surface Nonlinearity 8 493-515

Kolmogorov A N 1953 On dynamical systems with an integral invariant on the torus Dokl. Akad. Nauk SSSR $93763-6$ (in Russian)

Korteweg D 1899 Ueber eine ziemlich verbreitete unrichtige Behandlungsweise eines Problemes der rollenden Bewegung und insbesondere über kleine rollende Schwingungen um eine Gleichgewichtslage Nieuw Archiefvoor Wiskunde 4 130-55

Kozlov V V 1985 On the integration theory of the equations in nonholonomic mechanics Adv. Mech. 8 86-107

1988 Invariant measures of the Euler-Poincaré equations on Lie algebras Funct. Anal. Appl. 22 69-70

Marsden J E 1992 Lectures on Mechanics (London: Cambridge University Press)

Neimark Ju I and Fufaev N A 1972 Dynamics of Nonholonomic Systems Translations of Mathematical Monographs AMS 33

Rand R H and Ramani D V 2000 Relaxing nonholonomic constraints Proc. 1st Int. Symp. on Impact and Friction of Solids, Structues, and Intelligent Machines ed A Guran (Singapore: World Scientific) 113-6

Routh E J 1860 Treatise on the Dynamics of a System of Rigid Bodies. (London: MacMillan)

Schneider D A 2002 Nonholonomic Euler-Poincaré equations and stability in Chaplygin's sphere Dynamical Systems: An International J. 17 87-130

Suslov G K 1902 Theoretical Mechanics vol 2 (Kiev) (in Russian)

Veselov A P and Veselova L E 1988 Integrable nonholonomic systems on Lie groups Math. Notes 44 810-19

Vierkandt A 1892 Über gleitende und rollende Bewegung Monatshefte der Math. und Phys. III 31-54

Zenkov D V 1995 The geometry of the Routh problem J. Nonlinear Sci. 5 503-19

Zenkov D V, Bloch A M and Marsden J E 1998 The energy-momentum method for stability of nonholonomic systems Dyn. Stab. Syst. 13 123-65 